\title{
Chemotherapy and/or immune checkpoint inhibitors in NSCLC first-line setting: what is the best approach?
}

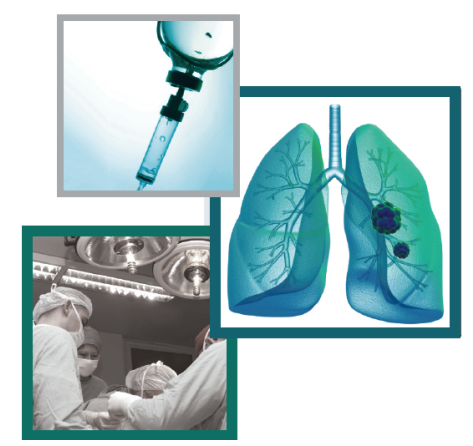

\author{
Luigi Della Gravara1', Ciro Battiloro², Rosa Cantile², Antonietta Letizia², Fabiana Vitiello², \\ Vincenzo Montesarchio 3 \& Danilo Rocco*,2 iD \\ ${ }^{1}$ Department of Experimental Medicine, Università degli Studi della Campania "Luigi Vanvitelli", Caserta, Italy \\ ${ }^{2}$ Division of Pulmonary Oncology, AORN dei Colli Monaldi, Naples, Italy \\ ${ }^{3}$ Division of Medical Oncology, AORN dei Colli Monaldi, Naples, Italy \\ *Author for correspondence: danilorocc@yahoo.it
}

'without a direct head-to-head comparison between pembrolizumab alone vs pembrolizumab + chemotherapy, the choice between these two regimes for the PD-L1 TPS $\geq 50 \%$ subset of patients must be made according to a case-to-case approach"

First draft submitted: 30 December 2019; Accepted for publication: 27 January 2020; Published online: 12 February 2020

Keywords: CheckMate $\bullet$ chemo-immunotherapy • first-line $\bullet$ immune checkpoint Inhibitors • IMpower $\bullet$ KEYNOTE - POSEIDON

Chemotherapy and immune checkpoint inhibitors (ICIs) exhibit a synergistic activity when administered together: by killing tumor cells, chemotherapy triggers the release of cancer antigens, thus enhancing cancer antigens presentation by antigen-presenting cells. Moreover, chemotherapy also presents an immunomodulatory role, both immune-stimulating and immune-suppressive. In fact, while it enhances the number of CD8+ tumor microenvironment (TME) infiltrating $\mathrm{T}$ cells and stimulates the maturation and activity of antigen-presenting cells, it also promotes down-regulation of myeloid-derived suppressor cells (MDSCs) and Tregs production and TME infiltration. Therefore, taking into account these preclinical and early clinical data, several chemo-immunotherapy combinations have been investigated in clinical settings and thanks to the very promising results achieved, to date, some of these combinations are US FDA and EMA approved and are starting to shape different international clinical guidelines.

Taking into account the existing preclinical and early clinical data regarding chemo-immunotherapy combinations [1], several of these have been investigated in clinical settings and thanks to the very impressive results achieved, to date, some of these combinations are US FDA and EMA approved and are starting to shape different international clinical guidelines [2]. In fact, as of January 2020, three chemo-immunotherapy combinations are FDA and EMA approved: cis/carboplatin + pemetrexed + pembrolizumab (nonsquamous NSCLC); carboplatin + paclitaxel/nab-paclitaxel + pembrolizumab (squamous NSCLC); and carboplatin + paclitaxel + bevacizumab + atezolizumab (nonsquamous NSCLC) [3-8]. Furthermore, several other chemo-immunotherapy associations - even though still neither FDA, nor EMA approved - have recently produced very promising data: carboplatin + nab-paclitaxel + atezolizumab (nonsquamous NSCLC); cis/carboplatin + pemetrexed + nivolumab + ipilimumab (nonsquamous NSCLC); cis/carboplatin + paclitaxel + nivolumab + ipilimumab (squamous NSCLC); cis/carboplatin + pemetrexed + durvalumab + tremelimumab (nonsquamous NSCLC); cis/carboplatin + gemcitabine + durvalumab + tremelimumab (squamous NSCLC); carboplatin + paclitaxel + durvalumab + tremelimumab (nonsquamous or squamous NSCLC; see Supplementary Table 1) [9-11].

\section{KEYNOTE-189}

The cis/carboplatin + pemetrexed + pembrolizumab combination was assessed in the KEYNOTE-189 trial, in which 616 naïve nonsquamous advanced NSCLC-affected patients without positive genetic biomarkers were randomized $(2: 1)$ to receive cis/carboplatin + pemetrexed + pembrolizumab followed by pembrolizumab and

Future $\because \%$ Medicine 
pemetrexed maintenance, or to receive cis/carboplatin + pemetrexed + placebo followed by placebo and pemetrexed maintenance [12]. As a result, the experimental arm outclassed the placebo one: median progression-free survival (PFS): 8.8 vs 4.9 months; hazard ratio (HR) for progression of disease (PD) or death: 0.52 ; with a comparable rate of grade 3-5 treatment-related adverse events (TRAEs) between the two arms: 67.2 vs $65.8 \%$. It is worth mentioning that these positive results were granted regardless of PD-L1 expression levels, however, a trend to better results was associated with increasing PD-L1 expression rates [13]. Moreover, following an updated analysis with a median follow-up of 18.7 months, overall survival (OS) data were disclosed, confirming the outstanding results of the combinational arm: 22.0 vs 10.7 months; HR for death: 0.56 .

\section{KEYNOTE-407}

The carboplatin + paclitaxel/nab-paclitaxel + pembrolizumab association was approved thanks to the results coming from the KEYNOTE-407 trial, in which 559 naïve squamous advanced NSCLC-affected patients without positive genetic biomarkers were randomized $(1: 1)$ to receive carboplatin + paclitaxel/nab-paclitaxel + pembrolizumab followed by pembrolizumab maintenance, or to receive carboplatin + paclitaxel/nab-paclitaxel + placebo followed by placebo maintenance [14]. The results clearly favored the experimental arm: median OS: 15.9 vs 11.3 months; HR for death: 0.64; median PFS: 6.4 vs 4.8 months; HR for PD or death: 0.56; with a comparable rate of grade 3-5 TRAEs between the two arms: 69.8 vs $68.2 \%$, respectively. Moreover, also in this trial the clinical benefit was observed independently from PD-L1 expression levels, although a trend to better results was associated with increasing PD-L1 expression rates.

\section{IMpower 150}

The carboplatin + paclitaxel + bevacizumab + atezolizumab was approved thanks to the data from the IMpower150 study, in which 1202 naïve nonsquamous advanced NSCLC-affected patients were randomized (1:1:1) to receive atezolizumab + carboplatin + paclitaxel + bevacizumab followed by bevacizumab and atezolizumab maintenance, or to receive atezolizumab + carboplatin + paclitaxel followed by atezolizumab maintenance, or to receive bevacizumab + carboplatin + paclitaxel followed by bevacizumab maintenance therapy $[15,16]$. The four drugs arm was then compared with the carboplatin + paclitaxel + bevacizumab one.

As a result, the experimental arm granted better results than the control one: median PFS: 8.3 vs 6.8 months; HR for PD or death : 0.62; median OS: 19.2 vs 14.7 months; HR for death: 0.78 ; with a comparable rate of grade 3-4 TRAEs between the two arms: 55.7 vs $47.7 \%$, respectively. The benefit in terms of survival was found to be independent from PD-L1 expression rates, even though better performances were associated to higher PD-L1 expression levels. Notably, the survival benefit was also granted in key subgroups, in other words, patients harboring EGFR mutations, $A L K$ rearrangements or liver metastases.

\section{IMpower 130}

In the IMpower130 study, 724 naïve nonsquamous advanced NSCLC-affected patients without positive genetic biomarkers were randomized (2:1) to receive carboplatin + nab-paclitaxel + atezolizumab, or site of care (SoC) chemotherapy followed by maintenance therapy [17]. Comparing these two treatments, collected data favored the former one: median overall survival (mOS): 18.6 vs 13.9 months (HR for death: 0.79); m PFS: 7 vs 5.5 months (HR for PD or death: 0.64) [9]. Although it is still neither FDA- nor EMA-approved, this combination recently received EMA's Committee for Medicinal Products for Human Use positive opinion for its implementation into clinical practice.

\section{CheckMate 9-LA}

On the other hand, in the CheckMate 9-LA trial, naïve nonsquamous or squamous advanced NSCLC-affected patients without positive genetic biomarkers were randomized to receive respectively cis/carboplatin + pemetrexed + nivolumab + ipilimumab vs SoC chemotherapy or cis/carboplatin + paclitaxel + nivolumab + ipilimumab vs SoC chemotherapy [10]. Even though the full dataset associated with this trial is still not disclosed, in a very recent press release, the Bristol-Myers Squibb Company (NY, USA) announced that these associations met their primary end point of superior OS at a prespecified interim analysis. However, these chemo-immunotherapy combinations are still neither FDA- nor EMA-approved. 


\section{POSEIDON}

In the same vein, in the POSEIDON study naïve advanced NSCLC-affected patients without positive genetic biomarkers were randomly assigned to receive cis/carboplatin + pemetrexed + durvalumab + tremelimumab, or carboplatin + paclitaxel + durvalumab + tremelimumab vs SoC chemotherapy (nonsquamous NSCLC) and cis/carboplatin + gemcitabine + durvalumab + tremelimumab, or carboplatin + paclitaxel + durvalumab + tremelimumab vs SoC chemotherapy (squamous NSCLC) [11]. Similar to CheckMate 9-LA, although the results of this trial are still not available, on 28 October 2019, AstraZeneca (MA, USA) announced that the chemotherapy + durvalumab + tremelimumab triple association met its primary end point of benefit in terms of PFS at a prespecified interim analysis. To date, however, this combination is still investigational.

\section{Chemo-immunotherapy combinations in the treatment of advanced NSCLC: different approaches for different patients?}

As the above-mentioned data show, chemotherapy plus immunotherapy combinations are currently starting to and will - in a very near future - replace chemotherapy as the standard of care treatment for most naïve nonsquamous or squamous advanced NSCLC-affected patients without positive genetic biomarkers, however, while the benefit is evident in patients expressing a PD-L1 tumor proportion score (TPS) $<50 \%$, it is not so clear in those with a PD-L1 TPS $\geq 50 \%$.

In fact, according to clinical international guidelines these patients are already eligible to receive first-line pembrolizumab instead of current standard of care chemotherapy.

To date there are no clinical data about a head-to-head comparison between first-line pembrolizumab vs first-line pembrolizumab + chemotherapy (presently offering the best outcomes among approved chemo-immunotherapy combinations) in naïve nonsquamous or squamous advanced NSCLC-affected patients without positive genetic biomarkers with a PD-L1 TPS $\geq 50 \%$, sparking a debate on the most effective and appropriate treatment.

On the one hand, some indirect comparison meta-analyses have been performed in order to answer this question, but due to their nature (i.e., no head-to-head comparisons) the results provided must be taken with caution. Doherty and colleagues constructed a meta-analysis including patients from the KEYNOTE-024 study and from the PD-L1 TPS $\geq 50 \%$ subgroups from the KEYNOTE-189 and 407 trials in order to compare pembrolizumab with pembrolizumab + chemotherapy [18]. The results showed no benefits in terms of OS, PFS or TRAEs; however, the pembrolizumab + chemotherapy combination was linked to higher overall response rates (ORR) and fewer early (first year of treatment) PFS events. Similarly, Zhou et al. designed a meta-analysis including patients from the KEYNOTE-024 study and from the PD-L1 TPS $\geq 50 \%$ subgroups from the KEYNOTE021 (cohort G), KEYNOTE-042, KEYNOTE-189 and KEYNOTE-407 trials, comparing pembrolizumab to pembrolizumab + chemotherapy [19]. These results showed that the pembrolizumab + chemotherapy association granted better results in terms of ORR and PFS, but no OS benefit was observed. Therefore, these results seem to suggest a sequential approach in this subset of patients, administering upfront single-agent pembrolizumab followed by post-progression classic standard of care chemotherapy. However, not only must we bear in mind the limited scope of these data, but we must also consider that a sequential approach could not be always a feasible option, in fact, according to the different estimates, up to $50 \%$ patients are not able to receive their intended second-line treatment due to the rapid functional and clinical post first-line-treatment progression of diseases, suggesting a the best first' approach [20].

Taking into account all the aforementioned data, it clearly appears that to date, without a direct head-tohead comparison between pembrolizumab alone vs pembrolizumab + chemotherapy, the choice between these two regimes for the PD-L1 TPS $\geq 50 \%$ subset of patients must be made according to a case-to-case approach, considering the patient's specific characteristics: for example, while a pembrolizumab monotherapy could better suit a patient with a low tumor burden and a better performance status, a pembrolizumab + chemotherapy combination would be more appropriate for a patient with a high tumor burden and a poor performance status, for whom a rapid and more probable response to treatment is crucial [21]. Moreover, this scenario is further complicated by the fact that patients expressing very high levels of PD-L1 TPS (i.e., 90-100\%) seem to represent a separate subgroup among patients expressing PD-L1 $\geq 50 \%$. For example, in a recent retrospective analysis Aguilar and colleagues compared ORR, median progression free survival and mOS outcomes in patients receiving first-line pembrolizumab expressing 90-100\% PD-L1 TPS to those expressing 50-89\% PD-L1 TPS [22]. As a result, all these outcomes were significantly improved in the former subgroup: ORR: 60 vs $32.7 \%$; median progression free survival: 14.5 vs 4.1 months (HR for PD or death: 0.50); mOS: not reached vs 15.9 months (HR for death: 0.39). 
Furthermore, this very same problem could soon arise with reference to atezolizumab, in fact, apart from the positive data coming from the IMpower 150 trial, atezolizumab also showed very interesting results in a recent interim analysis from the IMpower1 10 study. In this study, 555 high PD-L1 expressing (TC3/IC3) naïve nonsquamous or squamous advanced NSCLC-affected patients without positive genetic biomarkers were respectively randomized $(1: 1)$ to receive atezolizumab monotherapy vs cis/carboplatin + pemetrexed or atezolizumab monotherapy vs cis/carboplatin + gemcitabine and results decisively favored atezolizumab over SoC chemotherapy: mOS: 20.2 vs 13.1 months (HR for death: 0.595) [23].

Therefore, the quantification of PD-L1 tumor expression could represent highly helpful information, contributing to guide the diagnostic-therapeutic process.

\section{Supplementary data}

To view the supplementary data that accompany this paper please visit the journal website at: www.futuremedicine.com/doi/sup pl/10.2217//mt-2019-0018

Financial \& competing interests disclosure

C Gridelli has received honoraria as a speaker bureau and advisory board member for Merck Sharp \& Dohme, Bristol Myers Squibb, Roche, Astra Zeneca, Pfizer and Novartis. All the other authors have no relevant affiliations or financial involvement with any organization or entity with a financial interest in or financial conflict with the subject matter or materials discussed in the manuscript. This includes employment, consultancies, honoraria, stock ownership or options, expert testimony, grants or patents received or pending, or royalties.

No writing assistance was utilized in the production of this manuscript.

\section{Open access}

This work is licensed under the Attribution-NonCommercial-NoDerivatives 4.0 Unported License. To view a copy of this license, visit http://creativecommons.org/licenses/by-nc-nd/4.0/

\section{References}

1. Marshall HT, Djamgoz MBA. Immuno-oncology: emerging targets and combination therapies. Front. Oncol. 8, 315 (2018).

2. Rocco D, Della Gravara L, Battiloro C, Gridelli C. The role of combination chemo-immunotherapy in advanced non-small cell lung cancer. Expert Rev. Anticancer Ther. 19(7), 561-568 (2019).

3. US FDA. FDA grants regular approval for pembrolizumab in combination with chemotherapy for first-line treatment of metastatic nonsquamous NSCLC (2018). www.fda.gov/Drugs/InformationOnDrugs/ApprovedDrugs/ucm617471.htm

4. Merck \& Co., Inc. European commission approves Merck's KEYTRUDA ${ }^{\circledR}$ (pembrolizumab) in combination with pemetrexed and platinum chemotherapy for the first-line treatment of patients with metastatic nonsquamous NSCLC, with no EGFR or ALK genomic tumor aberrations (2018). https://investors.merck.com/news/press-release-details/2018/European-Commission-Approves-Mercks-KE YTRUDA-pembrolizumab-in-Combination-with-Pemetrexed-and-Platinum-Chemotherapy-for-the-First-Line-Treatment-of-Patients -with-Metastatic-Nonsquamous-NSCLC-with-No-EGFR-or-ALK-Genomic-Tumor-Aberrations/default.aspx

5. US FDA. FDA approves pembrolizumab in combination with chemotherapy for first-line treatment of metastatic squamous NSCLC (2018). www.fda.gov/Drugs/InformationOnDrugs/ApprovedDrugs/ucm624659.htm

6. Merck \& Co., Inc. European medicines agency adopts positive opinion for Merck's KEYTRUDA ${ }^{\circledR}$ (pembrolizumab) in combination with chemotherapy for first-line treatment of adults with metastatic squamous non-small cell lung cancer (NSCLC) (2019). https://investors.merck.com/news/press-release-details/2019/European-Medicines-Agency-Adopts-Positive-Opinion-for-Mercks-KEY TRUDA-pembrolizumab-in-Combination-with-Chemotherapy-for-First-Line-Treatment-of-Adults-with-Metastatic-Squamous-Non -Small-Cell-Lung-Cancer-NSCLC/default.aspx

7. US FDA. FDA approves atezolizumab with chemotherapy and bevacizumab for first-line treatment of metastatic non-squamous NSCLC (2018). www.fda.gov/Drugs/InformationOnDrugs/ApprovedDrugs/ucm627874.htm

8. Columbus G. Frontline atezolizumab regimen approved in Europe for NSCLC. OncLive, NJ, USA (2019). www.onclive.com/web-exclusives/f rontline-atezolizumab-regimen-approved-in-europe-for-nsclc

9. West H, McCleod M, Hussein M et al. Atezolizumab in combination with carboplatin plus nab-paclitaxel chemotherapy compared with chemotherapy alone as first-line treatment for metastatic non-squamous non-small-cell lung cancer (IMpower130): a multicentre, randomised, open-label, Phase III trial. Lancet Oncol. 20(7), 924-937 (2019).

10. Bristol-Myers Squibb. CheckMate -9LA, a Phase III trial evaluating opdivo (nivolumab) plus low-dose yervoy (ipilimumab) combined with chemotherapy, meets primary endpoint demonstrating superior overall survival compared to chemotherapy alone in first-line lung cancer. https://news.bms.com/press-release/corporatefinancial-news/checkmate-9la-phase-3-trial-evaluating-opdivo-nivolumab-plus-1 
11. AstraZeneca. Imfinzi and Imfinzi plus tremelimumab delayed disease progression in Phase III POSEIDON trial for 1st-line treatment of stage IV non-small cell lung cancer. www.astrazeneca.com/media-centre/press-releases/2019/imfinzi-and-imfinzi-plus-tremelimumab-d elayed-disease-progression-in-phase-iii-poseidon-trial-for-1st-line-treatment-of-stage-iv-non-small-cell-lung-cancer-html

12. Gadgeel SM, Garassino MC, Esteban E et al. KEYNOTE-189: updated OS and progression after the next line of therapy (PFS2) with pembrolizumab (pembro) plus chemo with pemetrexed and platinum vs placebo plus chemo for metastatic nonsquamous NSCLC. J. Clin. Oncol. 37(Suppl. 15), 9013-9013 (2019).

13. Gandhi L, Rodríguez-Abreu D, Gadgeel S et al. Pembrolizumab plus chemotherapy in metastatic non-small-cell lung cancer. $N$. Engl. J. Med. 378(22), 2078-2092 (2018).

14. Paz-Ares L, Luft A, Vicente D et al. Pembrolizumab plus chemotherapy for squamous non-small-cell lung cancer. N. Engl. J. Med. 379(21), 2040-2051 (2018).

15. Socinski MA, Jotte RM, Cappuzzo F et al. Atezolizumab for first-line treatment of metastatic nonsquamous NSCLC. N. Engl. J. Med. 378(24), 2288-2301.

16. Reck M, Mok TSK, Nishio M et al. Atezolizumab plus bevacizumab and chemotherapy in non-small-cell lung cancer (IMpower150): key subgroup analyses of patients with EGFR mutations or baseline liver metastases in a randomised, open-label Phase III trial. Lancet Respir Med. 7(5), 387-401 (2019).

17. Roche. European Commission approves Roche's new Tecentriq-based combination therapy as an initial treatment for most common form of advanced lung cancer. www.roche.com/media/releases/med-cor-2019-09-06.htm

18. Doherty M, Santos SD, Rahmadian AP et al. Pembrolizumab alone or with chemotherapy for PD-L1 positive NSCLC: A network meta-analysis of randomized trials. J. Clin. Oncol. 37(15_suppl), 9087-9087 (2019).

19. Zhou Y, Lin Z, Zhang X et al. First-line treatment for patients with advanced non-small cell lung carcinoma and high PD-L1 expression: pembrolizumab or pembrolizumab plus chemotherapy. J. Immunother. Cancer 7(1), 120 (2019).

20. Helwick C. Optimal first-line therapy for stage IV non-small cell lung cancer: immunotherapy alone or with chemotherapy? The ASCO Post (2019). www.ascopost.com/issues/august-25-2019/optimal-first-line-therapy-for-stage-iv-non-small-cell-lung-cancer/

21. Hellmann M, West H. Management of advanced non-small cell lung cancer lacking a driver mutation: immunotherapy (2019). www.uptodate.com/contents/management-of-advanced-non-small-cell-lung-cancer-lacking-a-driver-mutation-immunotherapy\#H19 76795012

22. Aguilar EJ, Ricciuti B, Gainor JF et al. Outcomes to first-line pembrolizumab in patients with non-small-cell lung cancer and very high PD-L1 expression. Ann. Oncol. 30(10), 1653-1659 (2019).

23. Roche. Roche's Tecentriq improves overall survival as a first-line monotherapy in certain people with advanced non-small cell lung cancer. www.roche.com/media/releases/med-cor-2019-09-27b.htm 